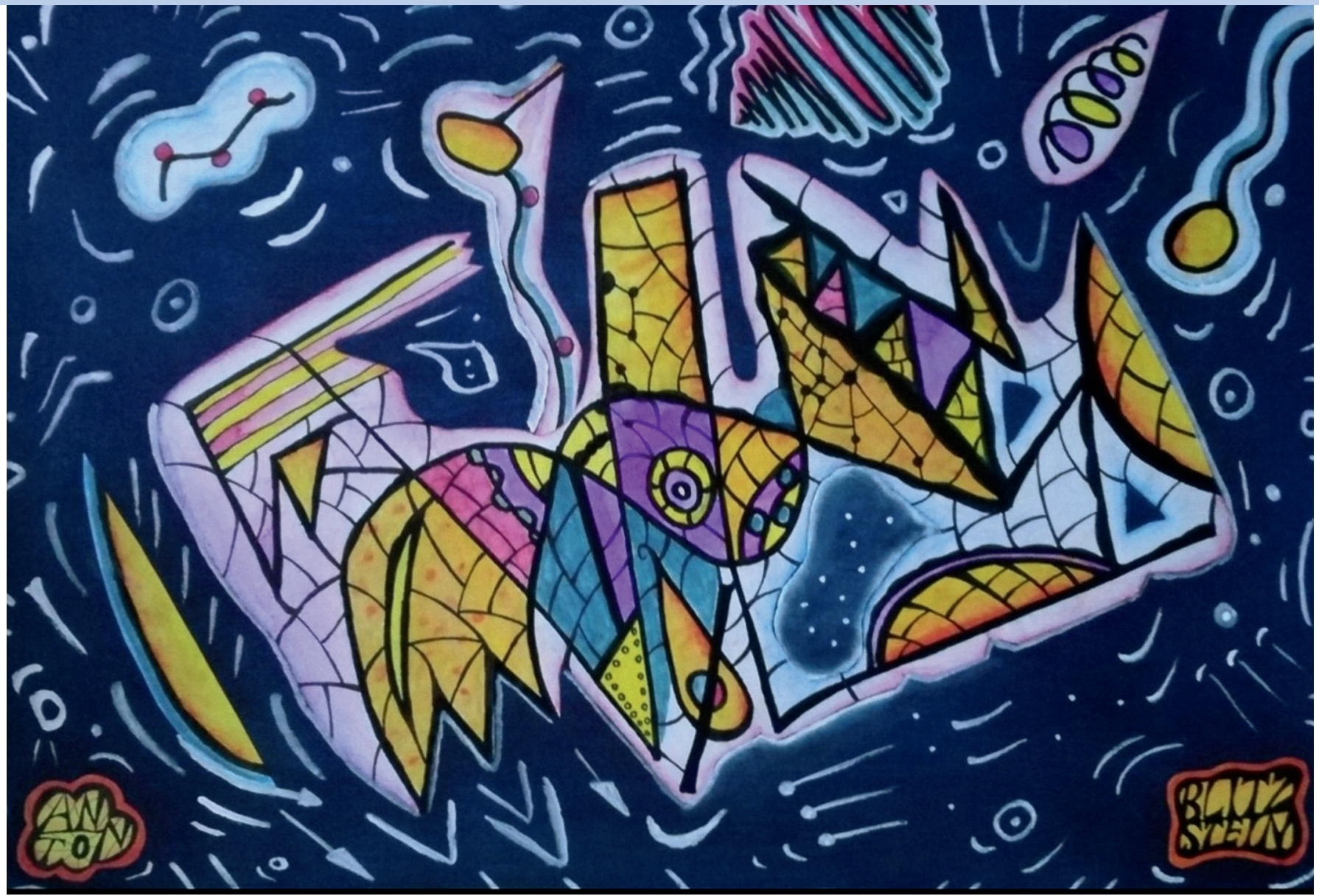

Anton Blitzstein, Gedankenströme

\title{
Postoperative pulmonale Komplikationen nach chirurgischen Eingriffen
}

Die Inzidenz postoperativer pulmonaler Komplikationen variiert je nach Art des chirurgischen Eingriffes und dem Patient*innenkollektiv. Unabhängig davon stellen pulmonale Komplikationen einen wesentlichen Faktor für die postoperative Mortalität dar. Während der chirurgische Eingriff sowie die Komorbiditäten der Patient*innen nicht veränderbar sind, gibt es einige perioperative Faktoren, die einen wesentlichen Einfluss auf die Entwicklung pulmonaler Komplikationen haben. Die Kenntnis dieser Faktoren sowie das Bewusstsein über Häufigkeit, Diagnose und Therapie ermöglichen eine bessere Betreuung der uns Anästhesist*innen anvertrauten Patient*innen.

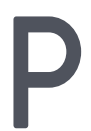
ostoperative pulmonale Kom-
plikationen (postoperative pul-
monary complication, PPC) treten häufig auf, erhöhen die Morbidität und Mortalität der Patient*innen und sind damit auch ein Kostenfaktor für das Gesundheitssystem. Die Ursachen sind mannigfaltig, doch ein Hauptaspekt ist der Eingriff in die Atemphysiologie durch eine Allgemeinanästhesie. Allein die Narkoseeinleitung führt zu einem schlagartigen Verlust des Atemantriebes sowie des Muskeltonus und damit zu einer Veränderung der Lungenvolumina. Die Anwendung von Muskelrelaxanzien aggraviert diesen Zustand und innerhalb kürzester Zeit treten Atelektasen bei einem

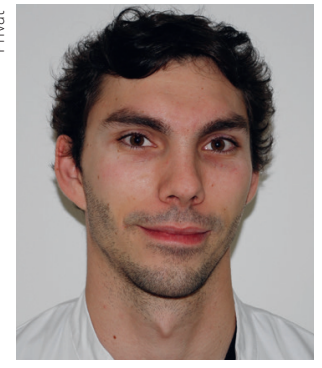

Von

Dr. Patrick

Spraider

Universitätsklinik

für Anästhesie

und Intensivmedizin MedUni Innsbruck

und

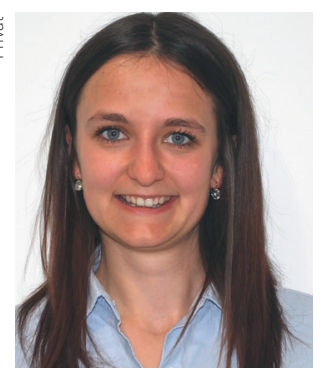

Großteil der Patient*innen auf. Die künstliche Überdruckbeatmung vermag die funktionelle Residualkapazität wiederherzustellen, doch führt sie global betrachtet auch zu einer erhöhten Energieeinwirkung auf die Lunge, die, sofern sie die lungenmechanischen Grenzen überschreitet, zu einer Ventilator-induzierten Lungenschädigung führt (ventilator induced lung injury, VILI). Moderne Beatmungskonzepte sind daher bestrebt, diese Schädigungen zu reduzieren. Dennoch sind selbst bei Patient*innen, bei denen regionalanästhesiologische Verfahren unter Erhalt der Spontanatmung durchgeführt werden, PPC zu beobachten. Daher kann die künstliche Beatmung 
nicht alleine für das Auftreten von PPC verantwortlich gemacht werden. Vielmehr gibt es zahlreiche weitere Einflussfaktoren wie etwa Immobilisation, nosokomiale Infektionen, erhöhter abdomineller Druck usw., die das Auftreten von PPC begünstigen.

\section{INZIDENZ}

Die Inzidenz von PPC variiert zum Teil sehr deutlich, was an dem untersuchten Kollektiv der Patient*innen und deren Komorbiditäten, dem Eingriff und auch maßgeblich an der Definition der PPC liegt.' Letzteres ist allerdings ein veränderbarer Umstand, dem verschiedene Fachgesellschaften Einhalt gebieten möchten, indem sie versuchen, eine Standarddefinition anzubieten, um eine bessere Vergleichbarkeit der einzelnen Studien zu ermöglichen. ${ }^{2,3}$ Zum Beispiel schwankt gerade bei risikobehafteten Eingriffen wie Herzoperationen die Inzidenz von PPC zwischen 10 und 50 Prozent, je nachdem, ob die Definition der Komplikation prolongierte Beatmung über 24 Stunden, Reintubationen, Pneumonien und ein schweres ARDS mit einer $\mathrm{PaO} 2 / \mathrm{FiO} 2$ Ratio unter 100 beinhaltet ${ }^{4}$ oder aber auch bereits die Notwendigkeit postoperativer NIV-Beatmung, die Anwendung von Bronchodilatatoren oder ein respiratorisches Versagen, definiert als Sauerstoffsättigung unter 90 Prozent bzw. PaO2/FiO2-Ratio unter 300 (siehe Tabelle). ${ }^{5}$

Aus den oben genannten Gründen schwankt die Inzidenz in der Literatur erheblich und wird mit zwischen 2 und 40 Prozent angegeben. ${ }^{1}$ GroBe thorakale Eingriffe wie Herz- oder Lungenoperationen haben die höchste Inzidenz mit etwa 40 Prozent, gefolgt von großen abdominellen Eingriffen wie Leber- oder Pankreaschirurgie mit 20 bis 40 Prozent. 6,7 Eingriffe ohne Eröffnung von Körperhöhlen wie etwa an den Extremitäten weisen die geringsten Inzidenzraten auf, die sich im einstelligen Prozentbereich bewegen. Rezente Untersuchungen ergaben eine globale PPC-Inzidenz von 2 bis 5 Prozent für elektive Operationen. ${ }^{8,9}$ Eine Datenanalyse von 1,2 Millionen chirurgischen Patient*innen bestätigt außerdem, dass von allen Komplikationen die pulmonalen die größte Reduktion an Lebensjahren verursachen, weshalb

\section{Tabelle: Definition der postoperativen pulmonalen Komplikationen nach ESA/ESICM ${ }^{2}$}

\begin{tabular}{|c|c|}
\hline Komplikation & Definition \\
\hline Respiratorische Infektion & $\begin{array}{l}\text { Patient*in erhielt eine antibiotische Therapie } \\
\text { aufgrund einer suszipierten Infektion und erfüllt } \\
\text { eines der folgenden Kriterien: neues oder } \\
\text { verändertes Sputum, neue oder veränderte } \\
\text { Trübung im Thorax-Röntgen, Fieber, Leuko- } \\
\text { zytenzahl über } 12 \times 10^{9 /-1}\end{array}$ \\
\hline Respiratorisches Versagen & $\begin{array}{l}\text { Postoperativer } \mathrm{PaO}_{2}<8 \mathrm{kPa}(60 \mathrm{mmHg}) \\
\text { unter Raumluft, } \mathrm{PaO}_{2} / \mathrm{FiO}_{2}-\text { Ratio }<40 \mathrm{kPa} \\
\text { (300 } \mathrm{mmHg} \text { ) oder } \mathrm{SpO}_{2}<90 \% \text { und } \\
\text { Notwendigkeit einer Sauerstoffgabe }\end{array}$ \\
\hline Pleuraerguss & Im Thorax-Röntgen sichtbar \\
\hline Atelektasen & $\begin{array}{l}\text { Eintrübung von Lungenabschnitten im Thorax- } \\
\text { Röntgen }\end{array}$ \\
\hline Pneumothorax & Im Thorax-Röntgen sichtbar \\
\hline Bronchospasmus & $\begin{array}{l}\text { Neu aufgetretenes exspiratorisches Giemen } \\
\text { unter Therapie mit Bronchodilatatoren }\end{array}$ \\
\hline Aspirationspneumonitis & $\begin{array}{l}\text { Akute respiratorische Insuffizienz nach } \\
\text { Aspiration von Mageninhalt }\end{array}$ \\
\hline
\end{tabular}

Die angeführte tabellarische Auflistung der postoperativen pulmonalen Komplikationen, definiert durch die Europäische Gesellschaft für Anästhesie (ESA) sowie die Europäische Gesellschaft für Intensivmedizin (ESICM), ermöglicht eine einheitlichere Aussage bezüglich postoperativer pulmonaler Komplikationen zur besseren Vergleichbarkeit der Studien.

die Autor*innen dieser Studie der Entwicklung von Strategien zur Senkung der PPC-Inzidenz große Bedeutung zur Verminderung der Gesamtmortalität attestieren. ${ }^{9}$

Anhand der geschilderten Daten ist ersichtlich, dass pulmonale Komplikationen ein häufig zu beobachtendes Ereignis darstellen und mit erheblichen Auswirkungen auf das Outcome der Patient*innen einhergehen.

\section{ASSOZIIERTE MORTALITÄT}

Die Inzidenz der PPC ist trotz einer hohen Schwankungsbreite unverkennbar bedeutsam, vor allem vor dem Hintergrund, dass das Auftreten von selbst milden pulmonalen Komplikationen mit einem Anstieg der Krankenhausaufenthaltsdauer und der postoperativen Mortalität assoziiert ist. ${ }^{10-12}$ In großen Multicenter-Studien konnte zum Beispiel bei Patient*innen mit auftretender PPC eine Krankenhausmortalität von 8 bis 10 Prozent und eine 30-TagesMortalität von 20 Prozent beobachtet werden. ${ }^{11,13,14}$ Zum Vergleich: Ein postoperativer Myokardschaden wies in einer Multicenter-Studie mit über 20.000 Patient*innen eine assoziierte 30-Tages-Mortalität von 4 Prozent auf. ${ }^{15}$ Pulmonale Komplikationen besitzen also nicht nur eine hohe Inzidenz, sondern stellen auch einen Hauptfaktor der perioperativen Mortalität dar.

Die Berücksichtigung der erhöhten PPC-Inzidenz ist von großer Relevanz, da präventive Konzepte wie etwa die Prähabilitation oder peri- und postoperative Therapien die Inzidenz nachweislich senken können und damit auch die Mortalität positiv beeinflusst wird. Es scheint daher sinnvoll, Konzepte zur Reduktion der perioperativen Mortalität bei risikobehafteten Operationen zu etablieren, wie sie in etwa in den ERAS-Richtlinien für Herzchirurgie ${ }^{16}$, Lungenchirurgie ${ }^{17}$ oder bei Ösophagusresektionen ${ }^{18}$ zu finden sind. Derartige Konzepte senken die Inzidenz der pulmonalen Komplikationen, wobei die Einbindung der chirurgischen Partner*innen einen wesentlichen Punkt darstellt. ${ }^{19}$ 


\section{PRÄOPERATIVE OPTIMIERUNG}

In der anästhesiologischen Prämedikationsvisite wird eine Nikotinkarenz für vier Wochen empfohlen, wobei die ideale Karenzzeit unbekannt ist, der größte Effekt jedoch nach vier Wochen beobachtet wurde. ${ }^{20}$ Einerseits ist die Raucher*innenanamnese allein als Risikofaktor identifiziert worden ${ }^{21}$, andererseits konnte auch gezeigt werden, dass in ihrer Leistungsfähigkeit nicht beeinträchtigte Raucher*innen kein erhöhtes Risiko für PPC aufweisen. ${ }^{13}$ Eventuelle Mangelernährungen sollten präoperativ ausgeglichen, eine vorbestehende Anämie sollte diagnostiziert und therapiert werden. Relevante pulmonale Vorerkrankungen betreffen das obstruktive Schlafapnoesyndrom (OSAS), die chronisch obstruktive Lungenerkrankung (chronic obstructive pulmonary disease, COPD), Asthma bronchiale sowie interstitielle Lungenerkrankungen. Während eine interstitielle Lungenerkrankung kaum veränderbar ist, wird bei vorbestehendem, symptomatischem Asthma bronchiale die Optimierung der medikamentösen Therapie empfohlen, da bei gut eingestellten Patient*innen kein erhöhtes Risiko zu beobachten ist. ${ }^{22}$ Ebenso ist das Stadium einer COPD kaum veränderbar, allerdings haben auch hier Studien gezeigt, dass die Diagnose allein keinen Risikofaktor darstellt, sondern vielmehr Narkosen zum Zeitpunkt einer Exazerbation vermieden werden sollten. ${ }^{13}$ Das betrifft auch pulmonale Infekte sowie Atemwegsinfekte, die aufgrund der Hyperreagibilität der Atemwege bzw. eingeschränkten Lungenfunktion die Inzidenz der PPC erhöhen. ${ }^{33}$ Wann eine elektive Operation nach durchgemachter SARSCoV-2-Infektion sicher ist, konnte bisher nicht eindeutig geklärt werden. Studien zeigten ein 2,8-fach erhöhtes Risiko für PPC bzw. ein 3,5-fach erhöhtes Mortalitätsrisiko bei einer Infektion innerhalb der letzten vier Wochen vor einem Eingriff, wobei bei asymptomatisch COVID-19-Erkrankten kein erhöhtes Risiko gefunden wurde. ${ }^{23}$ Dennoch wird eine siebenwöchige Verschiebung des Eingriffes nach Diagnose empfohlen $^{24}$, ebenso eine SARS-CoV-2-Impfung vor geplanten Eingriffen, da die Rate an nosokomialen Infektionen hoch ist $^{25}$ und eine perioperative COVID-19Erkrankung die Mortalität von 0,1 auf über 7 Prozent erhöht. ${ }^{26}$
Das OSAS stellt einen weiteren Risikofaktor für die Entwicklung von PPC dar ${ }^{27}$, hier gibt es aber präventive Möglichkeiten wie etwa die Anwendung kurzwirksamer Hypnotika, die Reduktion von Opiaten und eine postoperative prophylaktische NIV/CPAP-Therapie. Da ein OSAS häufig unterdiagnostiziert ist, spielt die Erkennung dieses Risikofaktors eine wesentliche Rolle, damit präventive Konzepte zur Anwendung kommen können. Dabei liegt der Fokus vor allem bei adipösen Patient*innen und bariatrischen Operationen, wo ein Screening auf das Vorliegen eines OSAS aufgrund der hohen Inzidenz sinnvoll erscheint. ${ }^{28}$

Körperliches Training im Sinne einer Prähabilitation als Vorbereitung für eine Operation ist eine multidisziplinäre Strategie, um das Outcome der Patien$t^{*}$ innen zu verbessern. Bisher wurde Prähabilitation vor allem bei Tumoroperationen untersucht. Es konnte gezeigt werden, dass eine Steigerung der präoperativen Leistungsfähigkeit zu einem besseren Outcome führt. ${ }^{29}$ Die Auswirkung auf die Rate an postoperativen pulmonalen Komplikationen ist heterogen, wobei sich ein Trend in Richtung positiver Effekte abzeichnet. ${ }^{29,30}$ Weitere randomisierte Studien sind notwendig, um einen klaren Effekt zu zeigen. Dabei ist anzumerken, dass vermutlich Patient*innen mit einem hohen Risikoprofil am meisten von Prähabilitationsprogrammen profitieren.

Weitere nicht beeinflussbare Risikofaktoren für das Auftreten von PPC sind das Alter, chirurgische Komplikationen oder die Notwendigkeit einer Massivtransfusion. Für die Anästhesie deutlich relevanter sind allerdings beeinflussbare operative Risikofaktoren, auf die behandelnde Ärzt*innen einen direkten Einfluss haben und die im folgenden Abschnitt erläutert werden.

\section{VENTILATION}

Ziel moderner Beatmungsstrategien ist es, das Auftreten von Ventilator-induzierten Lungenschäden zu verhindern. Besonders in einer Risikopopulation ist eine Lungen-protektive Beatmung ein wichtiger Faktor zur Verminderung der PPC-Inzidenz. ${ }^{31}$ Während die Anwendung einer sogenannten „low tidal volume ventilation" mit einem Tidalvo- lumen $(\mathrm{V} T$ ) von 6 bis $8 \mathrm{ml} / \mathrm{kg}$ idealem Körpergewicht und die Verwendung eines positiven endexspiratorischen Druckes (positive end-expiratory pressure, PEEP) weitgehend anerkannt sind ${ }^{32-35}$, werden die Wahl des idealen PEEP sowie die Verwendung von Rekrutierungsmanövern (RM) kontrovers diskutiert. Zwar konnten Studien, in denen eine individuelle PEEP-Titration durchgeführt wurde, eine Reduktion der benötigten Druckamplitude $(\Delta \mathrm{P})$ zeigen; die Inzidenz der PPC wurde dadurch allerdings nicht signifikant verändert. ${ }^{36}$ Eine große internationale Studie vergleicht derzeit die Verwendung eines hohen PEEP von $10 \mathrm{cmH}_{2} \mathrm{O}$ und die Anwendung von RM mit einem niedrigen PEEP von $5 \mathrm{cmH}_{2} \mathrm{O}$ ohne RM. ${ }^{37}$ Momentan gibt es jedoch keinen Anhaltspunkt für einen signifikanten Einfluss von PEEP oder RM auf die Inzidenz von PPC. ${ }^{38-40}$ Höhere PEEP-Werte und RM führen zwar zu einer verbesserten Lungen-Compliance und folglich zu einem reduzierten $\Delta P$; erhöhen jedoch zeitgleich auch Marker für epitheliale Lungenschädigung. ${ }^{41}$ Passend dazu konnte gezeigt werden, dass eine Erhöhung des PEEP, die zu einem Anstieg des $\triangle \mathrm{P}$ führt, die Inzidenz von postoperativen Komplikationen ansteigen lässt ${ }^{42}$ bzw. ein hoher PEEP von $12 \mathrm{cmH}_{2} \mathrm{O}$ auch negative hämodynamische Effekte wie vermehrte intraoperative Hypotension und einen höheren Vasopressorbedarf zur Folge hat, ohne die Rate an PPC zu reduzieren. ${ }^{39}$ Aus diesen Ergebnissen ist abzuleiten, dass der sicherste PEEP womöglich dem niedrigsten PEEP einer Compliancegesteuerten PEEP-Titration entsprechen könnte. Dieser kann bei einer Beatmung mit Volumenkonstanz wie etwa der volumenkontrollierten Beatmung (volume-controlled ventilation, VCV) dem PEEP-Wert gleichgesetzt werden, bei dem das geringste $\Delta P$ erforderlich ist. Bei einer Beatmung mit Druckkonstanz wie der druckkontrollierten Beatmung (pressure-controlled ventilation, PCV) entspricht dies dem PEEP-Wert, bei dem das größte Tidalvolumen bei identem $\Delta \mathrm{P}$ beobachtet werden kann. Eine solche Strategie berücksichtigt auch individuelle und operative Gegebenheiten und deren Auswirkungen auf die Lungenmechanik wie etwa Adipositas, COPD oder eine Laparoskopie mit Pneumoperitoneum, bei denen allesamt ein höherer PEEP vorteilhaft erscheint. 
Die Wahl des Beatmungsmodus ist ein weiterer Freiheitsgrad, der die Inzidenz der PPC beeinflusst. Studien konnten zeigen, dass die Verwendung einer VCV-Beatmung der PCV-Beatmung gegebenenfalls überlegen sein könn$\mathrm{te}^{42,43}$, da sie eine höhere Varianz der $V_{T}$ und $\Delta \mathrm{P}$ verhindert. Außerdem führt der hohe initiale Fluss bei PCV zu einer raschen Volumen- und Druckänderung in der Lunge, die den mechanischen Stress erhöht und zusätzlich eine präferenzielle Gasverteilung begünstigt. ${ }^{44}$ Ein neuer, viel diskutierter Aspekt ist die eingangs erwähnte mechanische Energie, die durch die Beatmung auf das Lungengewebe einwirkt. ${ }^{45,46}$ Das dezelerierende Flussprofil der druckkontrollierten Beatmung mit hohen Flussspitzen erhöht rein physikalisch die mechanische Energie, die auf das Lungengewebe wirkt. Notwendigerweise wird die einwirkende Energie auch mit der Verminderung des $\Delta \mathrm{P}$ reduziert, was für eine Compliancegesteuerte Beatmungseinstellung spricht. Mit der Berechnung der mechanischen Energie fließen aber erstmals auch die Atemfrequenz und damit der Faktor Zeit mit ein, der bei der alleinigen Betrachtung des $V_{T}$ und $\Delta \mathrm{P}$ keine Rolle gespielt hatte. Eine erste Studie, die $V_{T}, \Delta \mathrm{P}$ und mechanische Energie als mögliche Prädiktoren für Mortalität analysiert hat, konnte jedoch keinen Unterschied zwischen diesen drei Parametern finden. ${ }^{47}$

Ein neuer physikalischer Ansatz ist der Versuch einer Reduktion der Verlustleistung während der Beatmung. ${ }^{48,49}$ Dabei beschreibt die Verlustleistung die Energie, die während des Beatmungszyklus „verloren“ geht, entsprechend dem Energieerhaltungssatz von der Lunge absorbiert werden muss und potenziell zu Schäden führt. Zur Erreichung der geringsten Verlustleistung muss der Gasfluss während der Beatmung konstant und so gering wie möglich gewählt werden ${ }^{48}$, wozu ein neuer Beatmungsmodus, die flusskontrollierte Beatmung, entwickelt wurde. Diese ermöglicht nicht nur während der Inspiration einen konstanten Fluss (wie etwa auch bei der volumenkontrollierten Beatmung), sondern auch während der Exspiration, die erstmals aktiv kontrolliert wird. Studien konnten eine deutliche Steigerung der Beatmungs- effizienz hinsichtlich der Reduktion des Atemminutenvolumens und Steigerung der Oxygenierung zeigen ${ }^{50}$ sowie eine Verbesserung der Lungenbelüftung bei adipösen Patient*innen. ${ }^{51}$ Der Einfluss dieser neuen Beatmungsform auf PPC wird derzeit in klinischen Studien bei herz- und thoraxchirurgischen Eingriffen untersucht (ClinicalTrials.gov Identifier: NCT04306757, NCT04534933).

Um die schädigende Wirkung der mechanischen Ventilation zu minimieren, ist eine ehestmögliche Beendigung der künstlichen Beatmung anzustreben. So konnte gezeigt werden, dass selbst nach erfolgter Lebertransplantation eine sofortige Extubation möglich ist und diese die Inzidenz von PPC reduziert. ${ }^{52}$ Auch nach Herzoperationen ist die frühzeitige Extubation anzustreben $^{53-55}$, wobei hier meist eine Extubation < 6 Stunden postoperativ als frühzeitig bezeichnet wird, wenngleich selbst eine Extubation nach nur 1 bis 2 Stunden ${ }^{56,57}$ oder gar unmittelbar postoperativ möglich ist ${ }^{58}$ und bei entsprechender Spezialisierung sicher zu sein scheint.

\section{MUSKELRELAXANZIEN}

Die Anwendung von Muskelrelaxanzien ist einerseits eine notwendige Voraussetzung für die endotracheale Intubation, andererseits oft auch von chirurgischer Seite zur Verbesserung der Operationsbedingungen erwünscht. Außerdem erleichtert die Herabsetzung des Muskeltonus die künstliche Beatmung im Sinne einer Verbesserung der Lungen-Compliance. Es konnte allerdings gezeigt werden, dass die Anwendung von Muskelrelaxanzien die Bildung von Atelektasen begünstigt. In retrospektiven Studien ${ }^{59,60}$ konnte ein eindeutiger Zusammenhang zwischen dem Auftreten von PPC und der Verabreichung von Muskelrelaxanzien nachgewiesen werden, der in der europaweiten prospektiven Observationsstudie POPULAR bestätigt wurde. ${ }^{61}$ In keiner dieser Studien konnte ein signifikanter Einfluss einer Quantifizierung der Relaxierungstiefe mittels „Train-ofFour" (TOF) oder einer Reversierung mittels Neostigmin oder Sugammadex auf die Inzidenzrate von PPC gezeigt werden. In einer Post-hoc-Analyse der POPULAR-Studie ${ }^{62}$ konnte jedoch eine Reduktion der PPC-Inzidenz bei einem
TOF von > 95 Prozent vor Extubation festgestellt werden. Im Vordergrund steht dabei nicht die Behinderung der Atemmuskulatur, die unter einem TOF von 70 Prozent beobachtet wurde ${ }^{63}$, sondern die Aufrechterhaltung eines Muskeltonus im Pharynx zur Verhinderung der Atemwegsverlegung und der stillen Aspiration unmittelbar postoperativ. ${ }^{64}$ Diese Überlegung führte zu einer Kohorten-Untersuchung in der STRONGER-Studie, in der die Reversierung der Muskelrelaxation mittels Neostigmin versus Sugammadex untersucht wurde. ${ }^{65}$ Letzteres ermöglichte eine schnellere und wirksamere Reversierung im Vergleich zu Neostigmin und führte zu einer signifikanten Reduktion der PPC-Inzidenz. Dies konnte auch in einer Folgestudie (STIL-STRONGER) beobachtet werden. Allerdings konnte der Vorteil von Sugammadex in einer weiteren prospektiven, randomisierten Studie nicht bestätigt werden ${ }^{66}$ bzw. ergab auch eine weitere Observationsstudie widersprüchliche Ergebnisse. ${ }^{67}$ Dies könnte jedoch auch an der geringen Power der Studie liegen, da ein Trend zugunsten von Sugammadex sehr wohl zu beobachten war.

Zusammenfassend führt die Verabreichung von Muskelrelaxanzien zu einem Anstieg der PPC-Inzidenz, weshalb deren Anwendung kritisch hinterfragt werden sollte. Der Einfluss der Reversierung auf die Inzidenzrate und der möglicherweise positive Effekt von Sugammadex sind derzeit noch nicht hinreichend geklärt. Generell wird allerdings das Monitoring der Relaxierungstiefe mittels TOF stark empfohlen, wobei die derzeitige Evidenzlage auf einen Vorteil von > 95 Prozent Erholung gegenüber > 90 Prozent hindeutet. ${ }^{62}$

\section{PROPHYLAKTISCHE POSTOPERA- TIVE CPAP/NIV/NHF-THERAPIE}

Bei Patient*innen, die ein hohes Risiko für die Entwicklung postoperativer pulmonaler Komplikationen aufweisen - sei es aufgrund der Art des chirurgischen Eingriffes per se oder aufgrund ihrer Komorbiditäten -, kann eine prophylaktische postoperative Atemunterstützung mit kontinuierlichem Überdruck (CPAP) oder nichtinvasiver Beatmung (NIV) durchgeführt werden. Dabei hat sich in retrospektiven Studien ein Vorteil nach Herzoperationen gezeigt, in denen 


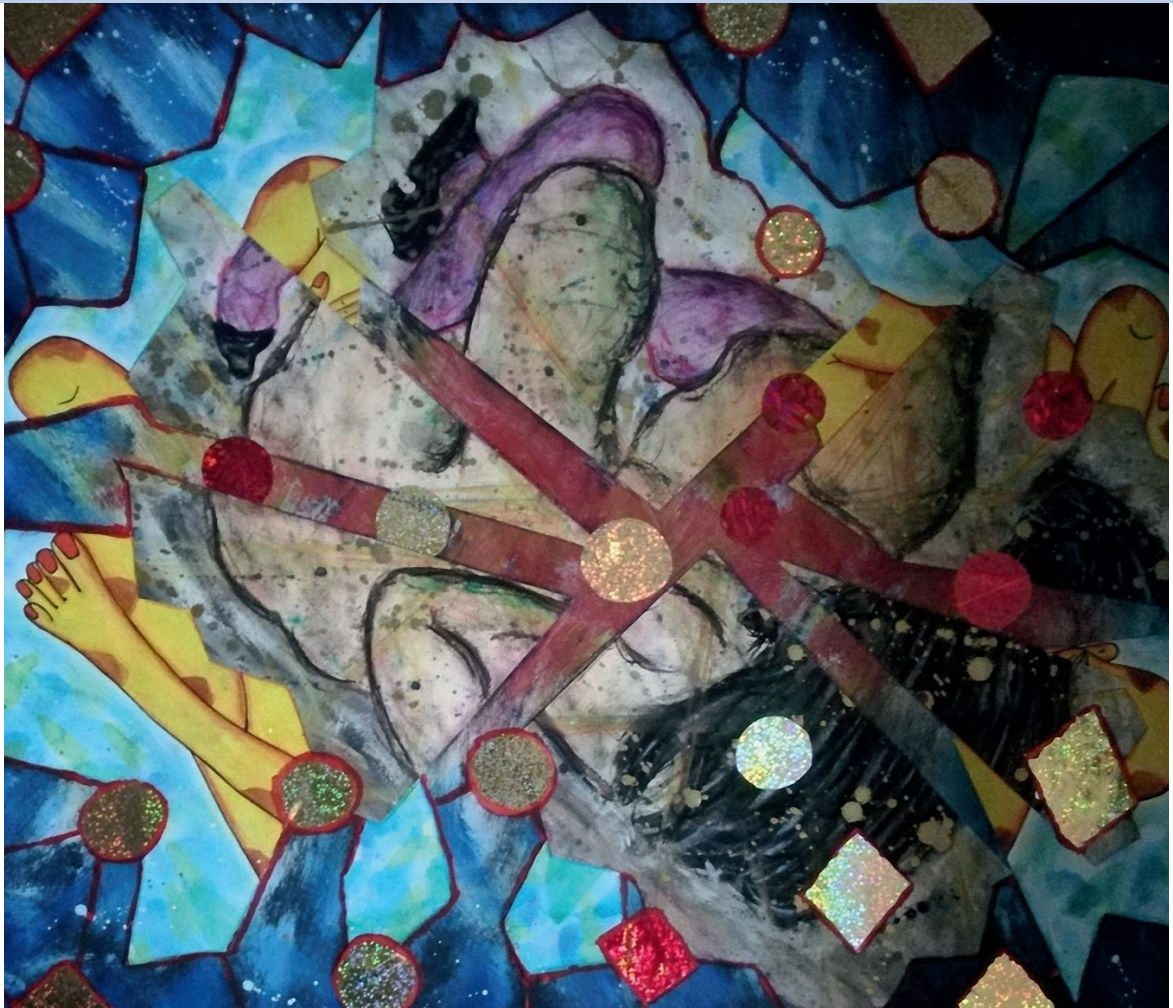

Anton Blitzstein, Der Farbenrausch

die Inzidenzraten der PPC durch die Anwendung von NIV reduziert werden konnten. ${ }^{68}$ Eine weitere Metaanalyse konnte zwar keine signifikante Reduktion der PPC-Inzidenz zeigen, beschrieb jedoch eine Reduktion des Intensivund Krankenhausaufenthaltes bei einer Verminderung der Atelektasen. ${ }^{69}$ Generell ist daher davon auszugehen, dass die prophylaktische Atemunterstützung postoperativ bei Hochrisikopatient*innen positive Effekte hat bzw. spätestens beim Auftreten von bereits mildem ARDS sinnvoll ist. Eine nasale High-Flow(nHF)-Sauerstofftherapie als Alternative zur reinen Sauerstoffgabe konnte die PPC-Inzidenz nicht senken ${ }^{70}$, wobei bei einer entsprechenden Risikopopulation die Reduktion der Reintubationsraten mittels $\mathrm{nHF}$ mit einer NIV-Therapie vergleichbar ist. ${ }^{71}$ Daher wird die prophylaktische nHF nur für Risikopatient*innen als Alternative zur CPAP/NIV-Therapie empfohlen. ${ }^{71}$ Es ist anzumerken, dass die konventionelle prophylaktische Sauerstoffgabe postoperativ risikobehaftet ist, da sie im Gegensatz zur CPAP-Therapie das Auftreten oder die Rückbildung von Atelektasen negativ beeinflusst und eine mögliche Hypoventilation aufgrund eines Sedativa-, Opiat- oder Muskelrelaxanzien-Überhangs bei häufig fehlender Überwachung des endtidalen oder arteriellen $\mathrm{CO}_{2}$-Gehaltes maskiert.

\section{Referenzen:}

1 Ball L et al. Curr Opin Crit Care 2016; 22(4):379. 385

2 Jammer I et al. Eur J Anaesthesiol 2015; 32(2):88 105

3 Abbott TEF et al. Br J Anaesth 2018; 120(5):1066

4 Mathis MR et al. Anesthesiology 2019; 131(5): 1046-1062

5 Liu Q et al. J Surg Res 2020; 246:384-394

6 Tsunoda S et al. Ann Surg Oncol 2021; 28(2):639 647

Karalapillai D et al. JAMA 2020; 324(9):848-858

8 COVIDSurg Collaborative; GlobalSurg Collaborative. Anaesthesia 2021; Epub ahead of print. doi: 10.1111/anae.15560.

9 Freundlich RE et al. Anesth Analg. 2018; 127(1):55 62

10 Fernandez-Bustamante A et al. JAMA Surg 2017 152(2):157-166

$11 \quad$ Canet J et al. Anesthesiology 2010; 113(6):1338-50

12 lbañez J et al. J Intensive Care Med 2016; 31(1):34 40

13 Mazo $\vee$ et al. Anesthesiology 2014; 121(2):219-31

14 Canet J et al. Eur J Anaesthesiol 2015; 32(7):458470

15 Writing Committee for the VISION Study Inves tigators, Devereaux PJ et al. JAMA; 317(16):1642 1651

16 Engelman DT et al. JAMA Surg 2019; 154(8):755 766

17 Batchelor TJP et al. Eur J Cardiothorac Surg 2019; 55(1):91-115

18 Low DE et al. World J Surg 2019; 43(2):299-330

19 Nicholson A et al. Br J Surg 2014; 101(3):172-88

20 Mills E et al. Am J Med 2011; 124(2):144-154 e8

21 Miskovic A et al. Br J Anaesth 2017; 118(3):317-334

22 Erdoğan T et al. Turk Thorac J 2020; 21(3):140-144

23 Kiyatkin ME et al. J Clin Anesth 2021; 74:110409

24 El-Boghdadly K et al. Anaesthesia 2021; 76(7):940 946

25 Read JM et al. Lancet 2021; S0140-6736(21)01786-4

26 Abbott TEF et al. Br J Anaesth 2021; 127(2): 205-214

27 Kaw R et al. Br J Anaesth 2012; 109(6):897-906

28 Members of the Working Party, Nightingale CE et al. Anaesthesia 2015; 70(7):859-876

29 Waterland JL et al. Front Surg 2021; 8:628848

30 Thomas G et al. Eur J Anaesthesiol 2019; 36(12): 933-945

31 LAS VEGAS investigators. Eur J Anaesthesiol 2017 34(8):492-507
32 Güldner A et al. Anesthesiology 2015; 123(3):692 713

33 Serpa Neto A et al. Lancet Respir Med 2014; 2(12):1007-1015

34 Serpa Neto A et al. Anesthesiology 2015; 123(1): 66-78

35 Ladha K et al. BMJ 2015; 351:h3646

36 Ferrando C et al. Lancet Respir Med 2018; 6(3): 193-203

37 Kiss T et al. Trials 2019; 20(1):213

38 Writing Committee for the PROBESE Collaborative Group of the PROtective VEntilation Network (PROVEnet) for the Clinical Trial Network of the European Society of Anaesthesiology, Bluth T, et al. JAMA 2019; 321(23):2292-2305

39 PROVE Network Investigators for the Clinical Trial Network of the European Society of Anaesthesiology, Hemmes SN et al. Lancet 2014; 384(9942):495-503

40 Lagier D et al. Intensive Care Med 2019; 45(10): 1401-1412

41 D'Antini D et al. Anesth Analg 2018; 126(1):143 149

42 Neto AS et al. Lancet Respir Med 2016; 4(4):272280

43 Bagchi A et al. Anaesthesia 2017; 72(11):1334-1343

44 Santini A et al. Crit Care 2019; 23(1):369

45 Gattinoni L et al. Intensive Care Med 2016; 42(10): 1567-1575

46 Silva PL et al. Intensive Care Med Exp 2019. 7(Suppl 1):38

47 Dianti J et al. Respir Care 2021; 66(2):221-227

48 Barnes T et al. Med Hypotheses 2018; 121:167-176

49 Marini JJ et al. Intensive Care Med 2021; 47(5): 609-611

50 Spraider P et al. Crit Care 2020; 24(1):662

51 Weber J et al. BMC Anesthesiol 2020; 20(1):24

52 Acho $C$ et al. Transplantation 2021; 105(9):2018 2028

53 García-Delgado $M$ et al. Curr Opin Anaesthesiol 2014; 27(2):146-152

54 Camp SL et al. J Card Surg 2009; 24(4):414-423

55 Camp SL et al. HSR Proc Intensive Care Cardio vasc Anesth 2009: 1(2):39-47

56 Probst S et al. Crit Care 2014; 18(4):468

57 Nicholson DJ et al. J Cardiothorac Vasc Anesth 2002; 16(1):27-31

58 Nagre AS et al. Ann Card Anaesth 2018; 21(2): 129-133

59 Bulka CM et al. Anesthesiology 2016; 125(4):647655

60 Grosse-Sundrup M et al. BMJ 2012; 345:e6329

61 Kirmeier E et al. Lancet Respir Med 2019; 7(2): 129-140

62 Blobner M et al. Br J Anaesth 2020; 124(1):63-72

63 Ali HH et al. Br J Anaesth 1975; 47(5):570-574

64 Sundman E et al. Anesthesiology 2000; 92(4): 977-984

65 Kheterpal S et al. Anesthesiology 2020; 132(6): 1371-1381

66 Togioka BM et al. Br J Anaesth 2020; 124(5):553 561

$67 \mathrm{Li} \mathrm{G}$ et al. Anesthesiology 2021; 134(6):862-873

68 Liu Q et al. J Surg Res 2020; 246:384-394

69 Wu Q et al. J Thorac Dis 2020; 12(4):1507-1519

70 Futier E et al. Intensive Care Med 2016; 42(12): 1888-1898

71 Chaudhuri D et al. Chest 2020; 158(5):1934-1946

\section{LECTURE BOARD:}

Prof. Dr. Thomas Fuchs-Buder Abteilung für Anästhesiologie und Intensivmedizin, Universität Lorraine, Nancy

\section{Dr. Thomas Hamp}

Universitätsklinik für Anästhesie, Allgemeine Intensivmedizin und Schmerztherapie, Medizinische Universität Wien/AKH Wien

\section{SPONSOR: MSD}

\section{FORTBILDUNGSANBIETER:}

Österreichische Gesellschaft für Anästhesiologie, Reanimation und Intensivmedizin 


\section{Postoperative pulmonale Komplikationen nach chirurgischen Eingriffen}

\author{
So machen Sie mit: \\ Entsprechend den Richt- \\ linien der ÖÄK finden Sie im \\ Anschluss an den Fortbil- \\ dungsartikel Multiple- \\ Choice-Fragen. Eine Frage \\ gilt dann als richtig beant- \\ wortet, wenn Sie von den \\ vorgegebenen Antworten \\ alle richtigen angekreuzt \\ haben. Für eine positive \\ Beantwortung ist erforder- \\ lich, dass Sie vier der sechs \\ Fragen richtig beantworten. \\ Bei korrekter Beantwortung \\ werden zwei DFP-Punkte \\ angerechnet.
}

\section{E-Mail, Post \& Fax:}

Schicken Sie diese Seite bitte per E-Mail an monica. friedmann@springer.at, per Post an Springer Medizin Wien (z. Hd. Monica Friedmann), Prinz-Eugen-Straße 8-10, 1040 Wien, oder per Fax an 01/330 2426.

Diesen Artikel sowie eine Reihe weiterer Fortbildungsangebote finden Sie auch auf www.pains. at und der Plattform „Akademie Lernwelt" der Österreichischen Akademie der Ärzte unter www.meindfp.at, wo Sie die Fragen auch online beantworten können.

Ihre Teilnahmebestätigung ist unter www.meindfp.at downloadbar, wenn Sie ein Fortbildungskonto haben.

Gültig bis: 10/2023
1. Welche Risikofaktoren sind für die Entwicklung postoperativer pulmonaler Komplikationen bekannt? (drei richtige Antworten)
a) Alter
b) Massivtransfusion
c) Depressio
d) Pulmonale Vorerkrankungen

2. Eine Lungen-protektive Beatmung entspricht ... (drei richtige Antworten)
a) ... einem Tidalvolumen von 6 bis $8 \mathrm{ml} / \mathrm{kg}$ idealem Körpergewicht.
b) ... der Vermeidung von hoher mechanischer Energie.
c) ... der Anwendung eines PEEP.
d) ... einem PEEP von mindestens $10 \mathrm{cmH}_{2} \mathrm{O}$.

3. Die Verabreichung von Muskelrelaxanzien ... (drei richtige Antworten)
a) ... erhöht die pulmonale Komplikationsrate.
b) ... sollte mittels „Train-of-Four" überwacht werden.
c) ... reduziert das Lungenvolumen nach Narkoseeinleitung.
d) ... sollte bevorzugt mit Neostigmin anstatt Sugammadex reversiert werden.

4. Eine postoperative CPAP/NIV-Therapie ... (drei richtige Antworten)
a) ... vermag die Intensivaufenthaltsdauer in einer Risikopopulation zu reduzieren.
b) ... sollte immer angewendet werden.
c) ... sollte bei auftretendem ARDS angewendet werden.
d) ... reduziert das Auftreten von Atelektasen.

5. Eine Prähabilitation ... (drei richtige Antworten)
a) ... ist vor allem bei Tumoroperationen untersucht.
b) ... verbessert die präoperative Leistungsfähigkeit.
c) ... reduziert deutlich die postoperative Mortalität.
d) ... erscheint bei erhöhtem Risiko für pulmonale Komplikationen sinnvoll.

6. Welche Faktoren haben einen NEGATIVEN Einfluss auf die Entwicklung postoperativer pulmonaler Komplikationen? (drei richtige Antworten)
a) Künstliche Überdruckbeatmung
b) Anwendung einer Regionalanästhesie
c) Verabreichung von Muskelrelaxanzien
d) Obstruktives Schlafapnoesyndrom

Name: 